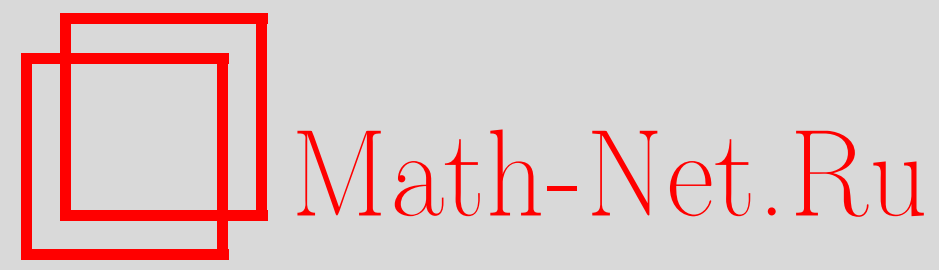

А. И. Ильинский, Г. П. Чистяков, К вопросу о характеризации вероятностных распределений абсолютными моментами частичных сумм, Теория вероятн. и ее примен., 1997, том 42, выпуск 3, 473-481

DOI: https://doi.org/10.4213/tvp1945

Использование Общероссийского математического портала Math-Net.Ru подразумевает, что вы прочитали и согласны с пользовательским соглашением http://www.mathnet.ru/rus/agreement

Параметры загрузки:

IP : 3.95 .254 .165

26 апреля 2023 г., 17:50:22

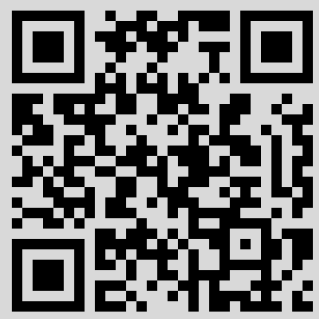




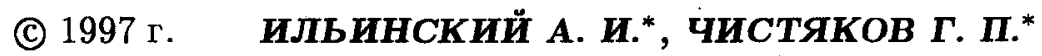

\title{
К ВОПРОСУ О ХАРАКТЕРИЗАЦИИ ВЕРОЯТНОСТНЫХ РАСПРЕДЕЛЕНИЙ АБСОЛЮТНЫМИ МОМЕНТАМИ ЧАСТИЧНЫХ СУММ')
}

\begin{abstract}
В работе указаны близкие к необходимым достаточные условия для однозначного определения симметричного вероятностного распределения по последовательности абсолютных моментов сумм независимых случайных величин с этим распределением.
\end{abstract}

Ключевые слова и фразы: характеристическая функция, суммы независимых случайных величин, абсолютные моменты, условие Крамера, интеграл типа Коши.

1. Введение и формулировка результатов. Пусть $F$ - вероятностное распределение (в.р.) на прямой с характеристической функцией (х.ф.) $\varphi(t ; F)$, обладаюшее конечным моментом первого порядка. Пусть $X_{1}, X_{2}, \ldots$ - независимые случайные величины с распределением $F$. При каждом натуральном $n$ положим

$$
a_{n}(F)=\mathbf{E}\left|\sum_{k=1}^{n} X_{k}\right|
$$

Через $F^{-}$обозначим распределение, определяемое следуюшим образом: $F^{-}(B)=F(-B)$ для всякого борелевского множества $B \subset \mathbf{R}$.

Как отмечено в $[1$, с. 270$]$, Л. Б. Клебанов поставил задачу нахождения условий на в.р. $F_{1}$ и $F_{2}$, выполнение которых обеспечивает справедливость импликащии

$$
a_{n}\left(F_{1}\right)=a_{n}\left(F_{2}\right), \quad n=1,2, \ldots \quad \Rightarrow \quad F_{1}=F_{2} .
$$

М. В. Неупокоева [2] доказала, что достаточно потребовать, чтобы в.р. $F_{j}$ были симметричными $\left(F_{j}^{-}=F_{j}, j=1,2\right)$ и обладали монотонно убываюшими на полуоси $(0, \infty)$ характеристическими функциями M. III. Браверман [3] показал, что достаточны такие условия: в.р. $F_{j}-$

*Физико-технический институт низких температур АН Украины, 310164 Харьков, Украина.

1) Работа выполнена при частичной финансовой поддержке гранта Сороса U9S200. 
симметричные, удовлетворяют условию Крамера

$$
\varlimsup_{t \rightarrow+\infty}\left|\varphi\left(t ; F_{j}\right)\right|<1
$$

х.ф. $\varphi\left(t ; F_{1}\right)$ аналитична в окрестности точки $t=0$. Причем, как показано в работе [1], условие (2) существенно. Там же приведены другие условия, достаточные для справедливости импликации (1).

В [1] сформулирован следуюший вопрос И. А. Ибрагимова: возможно ли, чтобы для двух разных распределений $F_{1}$ и $F_{2}\left(F_{1} \neq F_{2}, F_{1} \neq F_{2}^{-}\right)$ выполнялись условия $a_{n}\left(F_{1}\right)=a_{n}\left(F_{2}\right), n=1,2, \ldots$, и в то же время каждое из этих распределений удовлетворяло условию Крамера (2)? Там же высказано предположение о том, что пример таких распределений $F_{1}$ и $F_{2}$ невозможно построить, если дополнительно потребовать, чтобы $F_{1}$ и $F_{2}$ были симметричными. Мы покажем, что ответ на вопрос И. А. Ибрагимова положительный даже в классе симметричных в.p. (примеры 1 и 2, п. 3).

Мы также указываем новые условия, достаточные для справедливости импликации (1). Обозначим через $\mathcal{C}$ множество в.p. на $\mathbf{R} \mathbf{c}$ конечным моментом первого порядка, удовлетворяющих условию Крамера (2) и такому условию: для некоторого $b>0$ (зависящего от $F$ ) $\operatorname{Im} \varphi(t ; F)=0$ при $t \in[-b, b]$ и $\varphi(t ; F)$ строго монотонно убывает на отрезке $[0, b]$.

Пусть $D_{F, a}-$ замыкание множества $\{\varphi(t ; F): t \in \mathbf{R} \backslash(-a, a)\}$ в естественной топологии комплексной плоскости. Для $F \in \mathcal{C}$ положим:

$$
\begin{aligned}
& \alpha(F)=\sup \{a>0: \text { 1) } \operatorname{Im} \varphi(t ; F)=0, t \in[0, a] \\
& \text { 2) } \varphi(t ; F) \text { строго монотонно убывает на }[0, a] ; \\
& \text { 3) } \left.[\varphi(a ; F), 1] \cap D_{F, a}=\{\varphi(a ; F)\}\right\} .
\end{aligned}
$$

Обозначим через $U$ (соответственно, $U_{0}$ ) множество тех в.р. $F \in \mathcal{C}$, х.ф. $\varphi(t ; F)$ которых продолжается с отрезка $[-\alpha(F), \alpha(F)]$ (соответственно, с любого сколь угодно малого отрезка $[-a, a])$ на всю ось как положительно определенная функция единственным образом. Отметим, что $\mathcal{C} \supset U \supset U_{0}$. В класс $U_{0}$ входят симметричные в.р. с аналитичными в окрестности нуля х.ф., удовлетворяющими условию (2), но не исчерпывают его (см. пример 3, п. 3). Основной теоремой статьи является

Теорема 1. Если $F_{1}, F_{2} \in \mathcal{C}$ u $a_{n}\left(F_{1}\right)=a_{n}\left(F_{2}\right), n=1,2, \ldots$, mo $\varphi\left(t ; F_{1}\right)=\varphi\left(t ; F_{2}\right) n p u-\beta \leqslant t \leqslant \beta$, где $\beta=\min \left(\alpha\left(F_{1}\right), \alpha\left(F_{2}\right)\right)$.

Из этой теоремы получаются следуюшие условия, достаточные для справедливости импликации (1).

Теорема 2. Если $F_{1}, F_{2} \in U$, то выполняется (1).

Теорема 3. Если вероятностное распределение $F_{1} \in U_{0}$ и обладает конечным моментом порядка 2, а вероятностное распределение $F_{2}$ удовлетворяет условию Крамера (2) и его характеристическая 
Функция вещественна в нехоторой охрестности нуля, то выполняет$c . g(1)$.

3 а м е ч а и е 1 . Пусть $p>1$ и в.p. $F$ обладает конечным моментом порядка $p$. Положим $a_{n}^{(p)}(F)=\mathrm{E}\left|\sum_{k=1}^{n} X_{k}\right|^{p}, n=1,2, \ldots$. Можно поставить вопрос о нахождении условий, достаточных для справедливости импликации: $a_{n}^{(p)}\left(F_{1}\right)=a_{n}^{(p)}\left(F_{2}\right), n=1,2, \ldots \Longrightarrow F_{1}=F_{2}$ (см. [1]-[3]). Теоремы $1-3$ сохраняют силу в этом случае $(p \neq$ целому четному, $c p .[1])$. Доказательство в этом случае почти дословно повторяет доказательство для случая $p=1$, поэтому мы его не приводим.

3 а м е ч а н и е 2. Подчеркнем, что теоремы 2 и 3 (в отличие от теоремы 1) - это теоремы о симметричных распределениях, поскольку все в.р. классов $U$ и $U_{0}$ симметричны (так как если $\psi(t)-$ х.ф., то и $\operatorname{Re} \psi(t)-$ x.ф.).

2. Доказательства. Доказ а те ль с в о те о р м ы 1 . Будем опираться на следующую формулу, выражающую абсолютный момент случайной величины $X$ через ее х.ф. $\varphi(t)$ :

$$
\mathbf{E}|X|=\frac{2}{\pi} \int_{0}^{\infty} \frac{1-\operatorname{Re} \varphi(t)}{t^{2}} d t
$$

Пусть $F_{1}, F_{2}$ - в.р., удовлетворяющие условиям теоремы 1 . Для краткости будем писать $\varphi_{j}(t)$ вместо $\varphi\left(t ; F_{j}\right)$. Рассмотрим при комплексных $z,|z|>1$, функции $(j=1,2)$

$$
M_{j}(z)=\frac{1}{\pi} \int_{0}^{\infty}\left\{\frac{2}{z-1}-\frac{1}{z-\varphi_{j}(t)}-\frac{1}{z-\overline{\varphi_{j}(t)}}\right\} \frac{d t}{t^{2}} .
$$

Покажем, что функция $M_{j}(z)$ голоморфна в области $G_{j}$, являюшейся связной компонентой области $\mathbf{C} \backslash D_{F_{j}, 0}$, содержащей бесконечно удаленную точку. Действительно, для $z \in G_{j}$

$$
M_{j}(z)=\frac{2}{\pi} \frac{1}{z-1} \int_{0}^{\infty} \frac{\left(1-\operatorname{Re} \varphi_{j}(t)\right)(z+1)-\left(1-\left|\varphi_{j}(t)\right|^{2}\right)}{\left(z-\varphi_{j}(t)\right)\left(z-\overline{\varphi_{j}(t)}\right)} \frac{d t}{t^{2}}
$$

Поскольку $0 \leqslant 1-\left|\varphi_{j}(t)\right|^{2} \leqslant 1-\left(\operatorname{Re} \varphi_{j}(t)\right)^{2} \leqslant 2\left(1-\operatorname{Re} \varphi_{j}(t)\right)$, то в силу (3) и конечности первого момента в.p. $F_{j}$ имеем $\int_{0}^{\infty}\left(1-\left|\varphi_{j}(t)\right|^{2}\right) t^{-2} d t<\infty$. Поэтому интегралг в (5) сходится абсолютно и равномерно на любом компакте в $G_{j}$, откуда следует требуемое. Покажем теперь, что если выполнена посылка импликации (1), то $M_{1}(z)=M_{2}(z)$ при $z \in G_{1} \cap G_{2}$. При $|z|>1$ и $j=1,2$ в силу (3) имеем:

$$
\begin{aligned}
\frac{1}{z} \sum_{n=1}^{\infty} a_{n}\left(F_{j}\right) z^{-n} & =\frac{1}{z} \sum_{n=1}^{\infty} \frac{2}{\pi} \int_{0}^{\infty} \frac{1-\operatorname{Re} \varphi_{j}^{n}(t)}{z^{n} t^{2}} d t \\
& =\frac{2}{\pi z} \int_{0}^{\infty} \sum_{n=1}^{\infty} \frac{1-\operatorname{Re} \varphi_{j}^{n}(t)}{z^{n} t^{2}} d t
\end{aligned}
$$




$$
=\frac{1}{\pi} \int_{0}^{\infty}\left\{\frac{2}{z-1}-\frac{1}{z-\varphi_{j}(t)}-\frac{1}{z-\overline{\varphi_{j}(t)}}\right\} \frac{d t}{t^{2}}=M_{j}(z) .
$$

Почленное интегрирование ряда в (6) законно, поскольку

$$
\frac{1-\operatorname{Re} \varphi_{j}^{n}(t)}{t^{2}} \leqslant n^{2} \frac{1-\operatorname{Re} \varphi_{j}(t)}{t^{2}} \quad(t \in \mathbf{R})
$$

и $\sum_{n=1}^{\infty} n^{2}|z|^{-n}<\infty$ при $|z|>1$. Неравенство (7) можно обосновать следуюшим образом. Для любого комплексного числа $a$ такого, что $|a| \leqslant$ 1, имеем:

$$
\begin{aligned}
1-\operatorname{Re}\left(a^{n}\right)= & \operatorname{Re}\left[(1-a)\left(1+a+\cdots+a^{n-1}\right)\right] \\
= & \operatorname{Re}(1-a) \operatorname{Re}\left(1+a+\cdots+a^{n-1}\right) \\
& +\operatorname{Im} a\left(\operatorname{Im} a+\operatorname{Im} a^{2}+\cdots+\operatorname{Im} a^{n-1}\right) \\
\leqslant & (1-\operatorname{Re} a) n+|\operatorname{Im} a|^{2} \frac{n(n-1)}{2},
\end{aligned}
$$

так как $\left|\operatorname{Im} a^{k}\right| \leqslant k|\operatorname{Im} a|$. Применяя эту оценку с $a=\varphi_{j}(t)$ и пользуясь хорошо известным неравенством $\left|\operatorname{Im} \varphi_{j}(t)\right|^{2} \leqslant 2\left(1-\operatorname{Re} \varphi_{j}(t)\right)$, получим (7).

Поскольку $a_{n}\left(F_{1}\right)=a_{n}\left(F_{2}\right), n=1,2, \ldots$, то левые части (6) для $j=1,2$ совпадают при $|z|>1$. Поэтому $M_{1}(z)=M_{2}(z)$ при $|z|>1$, и, значит, при $z \in G_{1} \cap G_{2}$.

Разобьем интеграл в (4) на сумму двух интегралов, взятых по промежуткам $\left[0, \alpha_{j}\right]$ и $\left[\alpha_{j}, \infty\right)$. Поскольку $\varphi_{j}(t)=\bar{\varphi}_{j}(t)$ при $t \in\left[0, \alpha_{j}\right]$ (см. определения класса $\mathcal{C}$ и числа $\alpha_{j}=\alpha\left(F_{j}\right)$ ), то функцию $M_{j}(z)$ можно записать в виде

$$
M_{j}(z)=\frac{2}{\pi} \frac{1}{z-1} \int_{0}^{\alpha_{j}} \frac{1}{z-\varphi_{j}(t)} \frac{1-\varphi_{j}(t)}{t^{2}} d t+B_{j}(z)=A_{j}(z)+B_{j}(z)
$$

где функция $B_{j}(z)$ - голоморфна на интервале $\left(\varphi_{j}\left(\alpha_{j}\right), 1\right)$. Интеграл $A_{j}(z)$ преобразуем, сделав замену $u=\varphi_{j}(t)$. Обозначим $f_{j}(u)\left(\varphi_{j}\left(\alpha_{j}\right) \leqslant\right.$ $u \leqslant 1)$ функцию, обратную к функции $\varphi_{j}(t)$, рассматриваемой на отрезке $\left[0, \alpha_{j}\right]$. Поскольку $F_{j} \in \mathcal{C}$, то $f_{j}(u)$ корректно определена. Простые вычисления показывают, что

$$
A_{j}(z)=\frac{2}{\pi(z-1)} \int_{\varphi_{j}\left(\alpha_{j}\right)}^{1}(1-u) \frac{f_{j}^{\prime}(u)}{f_{j}^{2}(u)} \frac{d u}{u-z}
$$

Положим $\Delta=\max \left(\varphi_{1}\left(\alpha_{1}\right), \varphi_{2}\left(\alpha_{2}\right)\right)$. Тогда равенство $M_{1}(z)=M_{2}(z)$ ( $z \in G_{1} \cap G_{2}$ ) означает, что для $z=x+i y, \Delta<x<1$, и достаточно малых по модулю $y \neq 0$ выполняется $A_{1}(z)+B_{1}(z)=A_{2}(z)+B_{2}(z)$. Положим в этом равенстве сначала $z=x+i \varepsilon$, а потом $z=x-i \varepsilon$; из первого равенства вычтем второе и в полученном равенстве перейдем к пределу при $\varepsilon \downarrow 0$. В силу голоморфности функций $B_{j}(z)$ в точках 
интервала $(\Delta, 1)$ имеем: $B_{j}(x+i \varepsilon)-B_{j}(x-i \varepsilon) \longrightarrow 0(\varepsilon \downarrow 0), j=1,2$, $\Delta<x<1$. Далее воспользуемся следующим результатом. Tогда

Теорема А $[4$, с. 190]. Пусть $g(x)$ - суммируемая на $\mathbf{R}$ функция.

$$
\lim _{\varepsilon \rightarrow 0}\left\{\frac{1}{2 \pi i} \int_{-\infty}^{\infty} \frac{g(x) d x}{x-x_{0}-i \varepsilon}-\frac{1}{2 \pi i} \int_{-\infty}^{\infty} \frac{g(x) d x}{x-x_{0}+i \varepsilon}\right\}=g\left(x_{0}\right)
$$

для почти всех (по мере Лебега) точех $x_{0} \in \mathbf{R}$.

В силу теоремы А имеем: $A_{j}(x+i \varepsilon)-A_{j}(x-i \varepsilon) \longrightarrow-4 i f_{j}^{\prime}(x) / f_{j}^{2}(x)$ $(\varepsilon \downarrow 0)$ для почти всех $x \in(\Delta, 1)$. Поэтому $f_{1}^{\prime}(x) / f_{1}^{2}(x)=f_{2}^{\prime}(x) / f_{2}^{2}(x)$ для почти всех $x \in(\Delta, 1)$. Интегрируя это равенство по $x$ от $x_{0}$ до $x$, где $x_{0}, x \in(\Delta, 1)$, получим $1 / f_{1}(x)=1 / f_{2}(x)+C, x \in(\Delta, 1)$, где $C$ константа. Полагая $x=\varphi_{2}(t)$, получим, что при $t \in(0, \tilde{\beta})$

$$
\varphi_{2}(t)=\varphi_{1}\left(\frac{t}{1+C t}\right)
$$

где $\tilde{\beta}=\min \left(f_{1}(\Delta), f_{2}(\Delta)\right)$. Легко видеть, что тогда (8) выполняется при $t \in(0, \beta)$, где $\beta=\min \left(\alpha_{1}, \alpha_{2}\right)$. Теперь покажем, что постоянная $C$ в (8) равна 0 . Это следует из такой леммы.

Лемма 1. Пусть $G_{1}, G_{2} \in \mathcal{C}, a_{n}\left(G_{1}\right)=a_{n}\left(G_{2}\right)$ для бесконечно многих натуральньх $n$. Тогда существует последовательность $\left\{t_{k}\right\}$ такая, что $t_{k} \downarrow 0 u \varphi\left(t_{k}, G_{1}\right)=\varphi\left(t_{k}, G_{2}\right)$.

Д ок а 3 а те л ь с т в л е м м ы 1. Предположим, что утверждение леммы не имеет места. Тогда найдутся такое число $\gamma>0$ и такие в.р. $G_{1}$ и $G_{2} \in \mathcal{C}$, что для их х.ф. $\chi_{j}(t)=\varphi\left(t ; G_{j}\right)$ верно неравенство $\chi_{1}(t)<\chi_{2}(t)$ при $t \in(0, \gamma)$. Можно считать $\gamma>0$ столь малым, что $\chi_{j}(t)>0$ при $t \in(0, \gamma), j=1,2$. Обозначим через $d$ такое число, что $\sup _{t \geqslant \gamma}\left|\chi_{j}(t)\right| \leqslant d, j=1,2$. Поскольку выполняется условие Крамера (2), то $d<1$. Пусть $\tilde{\mathbf{N}}=\left\{n \in \mathbf{N}: a_{n}\left(G_{1}\right)=a_{n}\left(G_{2}\right)\right\}$. В силу условия леммы $\tilde{\mathbf{N}}$ - бесконечное множество и для $n \in \tilde{\mathbf{N}}$ интегралы $\int_{0}^{\infty} t^{-2}(1-$ $\left.\operatorname{Re} \chi_{j}^{n}(t)\right) d t$ одинаковы при $j=1$ и 2 . Значит,

$$
\int_{0}^{\gamma} t^{-2}\left(\chi_{2}^{n}(t)-\chi_{1}^{n}(t)\right) d t=\int_{\gamma}^{\infty} t^{-2} \operatorname{Re}\left(\chi_{1}^{n}(t)-\chi_{2}^{n}(t)\right) d t .
$$

Модуль интеграла, стоящего в правой части равенства (9), оценивается сверху величиной

$$
\int_{\gamma}^{\infty} t^{-2}\left(\left|\chi_{1}(t)\right|^{n}+\left|\chi_{2}(t)\right|^{n}\right) d t \leqslant \frac{2}{\gamma} d^{n}
$$

Оценим снизу интеграл, стоящий в левой части (9). Фиксируем число 
$\gamma_{1}$ такое, что $0<\gamma_{1}<\gamma$ и $\min _{0 \leqslant t \leqslant \gamma_{1}} \chi_{1}(t) \geqslant(d+1) / 2$. Тогда

$$
\begin{aligned}
\int_{0}^{\gamma} t^{-2}\left(\chi_{2}^{n}(t)-\chi_{1}^{n}(t)\right) d t & \geqslant \int_{0}^{\gamma_{1}} t^{-2} \chi_{1}^{n}(t)\left[\left(\frac{\chi_{2}(t)}{\chi_{1}(t)}\right)^{n}-1\right] d t \\
& \geqslant\left(\frac{d+1}{2}\right)^{n} n \int_{0}^{\gamma_{1}} t^{-2} \ln \frac{\chi_{2}(t)}{\chi_{1}(t)} d t
\end{aligned}
$$

Из (9)-(11) получаем

$$
0<\int_{0}^{\gamma_{1}}{ }^{-2} \ln \frac{\chi_{2}(t)}{\chi_{1}(t)} d t \leqslant \frac{2}{\gamma n}\left(\frac{2 d}{d+1}\right)^{n} \longrightarrow 0, \quad n \in \widetilde{\mathbf{N}}, \quad n \rightarrow \infty .
$$

Так как интеграл в (12) не зависит от $n$, то получаем противоречие, доказывающее справедливость леммы 1 .

3 а м е ч н и е 3 . Отметим, что в доказательстве леммы не использовалось условие монотонности х.ф. $\varphi\left(t ; G_{j}\right)$ в правой полуокрестности нуля.

Продолжим доказательство теоремы 1 . Так как х.ф. $\varphi_{j}(t), j=$ 1,2 , удовлетворяют всем условиям леммы, то существует последовательность $\left\{t_{k}\right\}, t_{k} \downarrow 0$, такая, что $\varphi_{1}\left(t_{k}\right)=\varphi_{1}\left(t_{k} /\left(1+C t_{k}\right)\right)$. В силу строгой монотонности функции $\varphi_{1}$ в правой полуокрестности нуля это возможно только при $C=0$. Итак, из (8) получаем: $\varphi_{1}(t)=\varphi_{2}(t)$ при $t \in[-\beta, \beta]$. Теорема 1 доказана.

3 а м е ч а и е 4. В случае $p>1$ ( $p \neq$ пелому четному) функции $M_{j}(z)$ надо определять следуюцим образом:

$$
\begin{aligned}
M_{j}(z)=\frac{1}{\pi} \int_{0}^{\infty} & \left\{\left.\sum_{k=0}^{[p]-1} \frac{t^{k}}{k !} \frac{d^{k}}{d t^{k}}\left(\frac{1}{z-\varphi_{j}(t)}+\frac{1}{z-\overline{\varphi_{j}(t)}}\right)\right|_{t=0}\right. \\
& \left.-\frac{1}{z-\varphi_{j}(t)}-\frac{1}{z-\overline{\varphi_{j}(t)}}\right\} \frac{d t}{t^{p+1}}
\end{aligned}
$$

Теорема 2 является очевидным следствием теоремы 1. Отметим, что теорема 2 содержит в себе результат М. В. Неупокоевой [2], цитированный выше, ибо если $\varphi\left(t ; F_{j}\right) \downarrow$ на $[0, \infty)$, то $\alpha\left(F_{j}\right)=+\infty$.

Доказательст во те оремы 3 . Пусть $\varphi_{j}(t)=\varphi\left(t ; F_{j}\right)$. В силу леммы 1 и замечания 3 существует такая последовательность $\left\{t_{k}\right\}$, $t_{k} \downarrow 0$, что $\varphi_{1}\left(t_{k}\right)=\varphi_{2}\left(t_{k}\right)$. Отсюда следует, что $1-\varphi_{2}\left(t_{k}\right)=O\left(t_{k}^{2}\right)$, поскольку, в силу условия теоремы, $1-\varphi_{1}\left(t_{k}\right)=O\left(t_{k}^{2}\right)$. Значит, в.р. $F_{2}$ обладает конечным моментом порядка 2. Так как $F_{2}$ - невырожденное B.p., то $\varphi_{2}(t)$ строго монотонно убывает в правой полуокрестности нуля. Значит, $F_{2} \in \mathcal{C}$. В силу теоремы 1 отсюда следует, что $F_{1}=F_{2}$.

Отметим, что теорема 3 содержит в себе результат М. ШІ. Бравермана [3], цитированный во введении. Действительно, нужно только заметить, что если вещественная х.ф. $\varphi(t), \varphi(t) \not \equiv 1$, дважды дифференцируема в нуле, то она строго монотонно убывает в некоторой правой 
полуокрестности нуля. Единственность продолжения $\varphi(t)$ с любого отрезка, содержашего нуль, на всю ось как положительно определенной функции следует из аналитичности $\varphi(t)$ в окрестности нуля.

3 а м е ч а и е 5 . Легко видеть, что в теореме 3 требование конечности момента порядка 2 можно наложить на $F_{2}$, а не на $F_{1}$.

3. Примеры. П р и м е $\mathrm{p}$ 1. Для любых натурального числа $m$ и положительного числа $A$ существуют в.р. $F_{1}$ и $F_{2}$ такие, что

1) $F_{1}$ и $F_{2}$ обладают конечными моментами до порядка $m$ включительно,

2) $F_{1} \neq F_{2}, F_{1} \neq F_{2}^{-}$,

3) $a_{n}\left(F_{1}\right)=a_{n}\left(F_{2}\right)$ при всех $n \in \mathbf{N}$,

4) $\varphi\left(t ; F_{j}\right)=0$ при $|t| \geqslant A(j=1,2)$.

П р и м е $\mathrm{p} 2$. Для любых натурального $m$ и положительного $A$ существуют в.р. $F_{1}$ и $F_{2}$, удовлетворяющие условиям 1)-4) примера 1 , а также условию

5) $F_{j}=F_{j}^{-}(j=1,2)$.

Конечно, ответ на сформулированные во введении вопросы дает и один пример 2. Мы приводим два примера, так как при их построении используются разные соображения. Построение обоих примеров основывается на следующей лемме.

Лемма 2. Пусть $\chi(t)$ - характеристическая функиия, удовлетворяющая условиям:

А) плотность соответствуючего распределения $p_{\chi}(x)$ непрерьвна и $p_{\chi}(x)>0, \forall x \in \mathbf{R}$;

Б) существуют натуральное $N>1$ и положительное $x_{0}$ такие, что $p_{\chi}(x) \geqslant D|x|^{-N}$ при $|x| \geqslant x_{0}$, аде $D$ - положительная постоянная.

Пусть $\gamma(t)$ - комплекснозначная функция на $\mathbf{R}$, удовлетворяющая условиям:

a) $\gamma \in C^{N}(\mathbf{R})$, ade $N-$ mo же, чmo в $\left.\mathrm{b}\right)$;

б) $\gamma(-t)=\bar{\gamma}(t)$ npu всеx $t \in \mathbf{R}, \gamma(0)=0$;

в) $\gamma^{(k)}(t) \in L^{1}(\mathbf{R}) u \gamma^{(k)}(t) \rightarrow 0 n p u t \rightarrow \pm \infty, k=0,1,2, \ldots, N$.

Tогда суиествует такое $\varepsilon_{0}>0$, ито для всех $\varepsilon \in\left[-\varepsilon_{0}, \varepsilon_{0}\right]$ функчия $\varphi(t)=\chi(t)+\varepsilon \gamma(t)$ является характеристической функцией.

Д о к а за те ль с т о л л м ы 2. Надо убедиться в сушествовании $\varepsilon_{0}>0$ такого, что при всех $\varepsilon \in\left[-\varepsilon_{0}, \varepsilon_{0}\right]$ выполняется $p_{\varphi}(x)=$ $(2 \pi)^{-1} \int_{-\infty}^{\infty} \exp (-i t x) \varphi(t) d t \geqslant 0$ для всех $x \in \mathbf{R}$. Имеем $p_{\varphi}(x)=p_{\chi}(x)+$ $\varepsilon p_{\gamma}(x)$, причем $p_{\gamma}(x)$ - вещественнозначная функция. Интегрирование по частям показывает, что $\left|p_{\gamma}(x)\right|=O\left(|x|^{-N}\right), x \rightarrow \pm \infty$. Учитывая непрерывность функций $p_{\chi}(x)$ и $p_{\gamma}(x)$, убеждаемся в существовании требуемого $\varepsilon_{0}$.

Переходим к построению примера 1 . Обозначим $k$ такое натуральное число, что $2 k \geqslant m+2$. Положим $a=A /(4 k)$. Пусть $\theta(t)=(1-t / a)^{2}$ при $0 \leqslant t \leqslant a, \theta(t)=0$ при $t>a, \theta(t)=\theta(-t)$ при $t<0$. Функщия $\theta(t)$ 
является х.ф. в.р. с плотностью $p_{\theta}(x)=2(\pi a)^{-1} x^{-2}\left(1-(a x)^{-1} \sin a x\right)$ при $x \neq 0, p_{\theta}(0)=a(3 \pi)^{-1}$. Заметим, что $p_{\theta}(x)>0$ при всех $x \in \mathbf{R}$ и $p_{\theta}(x) \sim 2(\pi a)^{-1} x^{-2}$ при $x \rightarrow \pm \infty$. Положим $\chi(t)=C \theta^{k *}(t)$, где $\theta^{k *}(t)$ есть $k$-кратная свертка функции $\theta(t)$ с собой, а $C$ - такое положительное число, что $\chi(0)=1$. Тогда $\chi(t)-\mathrm{x} . ф$. плотности $p_{\chi}(x)=C(2 \pi)^{k-1} p_{\theta}^{k}(x)$, причем выполняются условия А), Б) леммы 2 с $N=2 k$. Заметим, что $\chi(t)>0$ при $|t|<A / 2, \chi(t)=0$ при $|t| \geqslant A / 2$.

Пусть $\rho(t)$ - произвольная функция, удовлетворяюшая условиям: $\rho(t) \in C^{\infty}(\mathbf{R}), \rho(t)>0$ при $t \in(0, A / 4), \rho(t)=0$ при $t \notin(0, A / 4)$. Пусть $\alpha \in \mathbf{R}$ и $\alpha \neq 0(\bmod \pi)$. Положим для $j=1,2$

$$
\gamma_{j}(t)= \begin{cases}e^{i \alpha} \rho\left(t-\frac{A}{2}\right), & t \in\left[\frac{A}{2}, \frac{3 A}{4}\right] \\ \exp \left((-1)^{j} i \alpha\right) \rho\left(t-\frac{3 A}{4}\right), & t \in\left[\frac{3 A}{4}, A\right] \\ 0, & t \in\left[0, \frac{A}{2}\right] \bigcup[A, \infty), \\ \bar{\gamma}_{j}(-t), & t \in(-\infty, 0)\end{cases}
$$

Функции $\gamma_{j}(t)$ удовлетворяют условиям a)-в) леммы 2. Поэтому при всяком достаточно малом по модулю вещественном $\varepsilon$ функции $\varphi_{j}(t)=$ $\chi(t)+\varepsilon \gamma_{j}(t)$ будут характеристическими функциями. Обозначим через $F_{j}$ такие в.р., что $\varphi\left(t ; F_{j}\right)=\varphi_{j}(t), j=1,2$. Тогда утверждения 1$), 2$ ), 4) примера 1 выполняются очевидным образом. Справедливость утверждения 3) вытекает из формулы (3) и из того, что $\operatorname{Re} \varphi_{1}^{n}(t) \equiv \operatorname{Re} \varphi_{2}^{n}(t)$ при всех $n \in \mathbf{N}$.

Приведем построение примера 2. Пусть $\chi(t)$ - х.ф. из примера 1. Пусть функция $r(u)$ удовлетворяет условиям: $r(u) \in C^{\infty}(\mathbf{R}), r(u)>0$ при $u \in(0,1 /(2 A)), r(u)=0$ при $u \notin(0,1 /(2 A))$. Определим функции $r_{j}(u), j=1,2$, так:

$$
r_{1}(u)=\left\{\begin{array}{ll}
r\left(u-\frac{1}{A}\right), & u \in\left[\frac{1}{A}, \frac{3}{2 A}\right] \\
-r\left(u-\frac{3}{2 A}\right), & u \in\left[\frac{3}{2 A}, \frac{2}{A}\right], \\
0, & u \in\left[0, \frac{1}{A}\right] \bigcup\left[\frac{2}{A}, \infty\right], \\
r_{1}(-u), & u \in(-\infty, 0) .
\end{array} \quad r_{2}(u)=-r_{1}(u) .\right.
$$

Положим $\gamma_{j}(t)=r_{j}(1 / t), \varphi_{j}(t)=\chi(t)+\varepsilon \gamma_{j}(t)$. Применяя лемму 2, видим, что функции $\varphi_{j}(t)$ являются х.ф. при вещественном $\varepsilon$, достаточно малом по модулю. Пусть $F_{1}$ и $F_{2}$ - распределения с х.ф. $\varphi_{1}(t)$ и $\varphi_{2}(t)$ соответственно. Выполнение утверждений 1), 2), 4), 5) для таких распределений очевидно. Справедливость утверждения 3) вытекает из того, 
что при всех $n \in \mathbf{N}$

$$
\begin{aligned}
a_{n}\left(F_{j}\right)= & \frac{2}{\pi} \int_{0}^{\infty} \frac{1-\varphi_{j}^{n}(t)}{t^{2}} d t \\
= & \frac{2}{\pi} \int_{0}^{A / 2} \frac{1-\chi^{n}(t)}{t^{2}} d t+\frac{4}{\pi A}+\frac{2 \varepsilon^{n}}{\pi} \int_{1 / A}^{2 / A} r_{j}^{n}(u) d u \\
& \int_{1 / A}^{2 / A} r_{1}^{n}(u) d u=\int_{1 / A}^{2 / A} r_{2}^{n}(u) d u
\end{aligned}
$$

П р и м е р 3. Приведем пेримеры в.р. из класса $U_{0}$ с неаналитическими в точке 0 характеристическими функциями. Такие в.р. фигурируют в теореме 3. Нам понадобится результат, отмеченный в [5, с. 215], который является следствием одного результата, доказанного П. Куси$\operatorname{com}[6$, c. 236$]$.

Теорема Б. Пусть $\left\{\lambda_{n}\right\},\left\{l_{n}\right\}-$ две последовательности положительных чисел, $\lambda_{n} \rightarrow+\infty(n \rightarrow \infty)$ и промежутки $\left(\lambda_{n}, \lambda_{n}+l_{n}\right)$ попарно не пересекаются. Пусть вероятностное распределение $F$ сосредоточено на множестве $\mathbf{R} \backslash \cup_{n=1}^{\infty}\left(\lambda_{n}, \lambda_{n}+l_{n}\right)$. Eсли выполняется условие $\sum_{n=1}^{\infty}\left(l_{n} / \lambda_{n}\right)^{2}=\infty$, то характеристическап функиия $\varphi(t ; F)$ допускает единственное продолжение с каждого конечного интервала $(-l, l)$, $l>0$.

Эта теорема позволяет легко привести примеры симметричных в.р. $F \in U_{0}$ с бесконечным третьим моментом. Положим для каждого нату: рального $n$ : $\lambda_{n}=e^{n}, l_{n}=e^{n-1} / \sqrt{n}$. Обозначим $E=\mathbf{R} \backslash \cup_{n=1}^{\infty}\left\{\left(\lambda_{n}, \lambda_{n}+\right.\right.$ $\left.\left.l_{n}\right) \cup\left(-\lambda_{n}-l_{n},-\lambda_{n}\right)\right\}$. Пусть $F-$ в.p. с плотностью, равной $C|x|^{-7 / 2}$ при $x \in E$ и нулю при $x \notin E$. Легко проверить, что момент порядка 2 в.р. $F$ конечен, а момент порядка 3 бесконечен. $F \in U_{0}$ в силу теоремы Б.

\section{СПИСОК ЛИТЕРАТУРЫ}

1. Бравермая M. III., Маллоус K. Л., IIIепn Л. А. Характеризация вероятностных распределений абсолютными моментами частичных сумм. - Теория вероятн. и ее примен., 1995, т. 40, в. 2, с. 270-285.

2. Неупокоева $M$. B. О восстановлении распределения по моментам сумм независимых случайных величин. - В сб.: Проблемы устойчивости стохастических моделей: Тр. семинара. М.: ВНИИСИ, 1989, с. 104-108.

3. Braverman $M$. Sh. A characterization of probability distributions by moments of sums of independent random variables. - J. Theor. Probab., 1994, v. 7, № 1, p. 187-198.

4. Привалов И. Н. Граничные свойства аналитических функций. М.-Л.: Гостехиздат, 1950, 336 c.

5. Качнельсон $B$. Э. Методы $J$-теории в континуальных интерполяционных задачах анализа. Ч. І. Харьков, 1982, 249 с. ЦИОНТ ПИК ВИНИТИ № 39.

6. Koosis $P$. The Logarithmic Integral. I. Cambridge: Cambridge Univ. Press, 1988, $606 \mathrm{p}$. 